\title{
PRESAGE (PREparation de Surface et nettoyAGE par laser) applications aéronautiques : nettoyage de moules d'injection et préparation avant collage de matériaux composites
}

\author{
B. Esmiller
}

Aérospatiale, Département Techniques de Base Physique et Mathématiques, Service Optique, Laser et Thermique, Centre de Recherches Louis Blériot, 12 rue Pasteur, 92152 Suresnes cedex, France

\begin{abstract}
Résumé : Cet article présente la démarche suivie dans le cadre du projet innovant PRESAGE (PREparation de Surface et nettoyAGE par laser) pour permettre aux différents partenaires industriels, avec l'aide de centres de recherches et de transfert de technologie, d'évaluer, techniquement et économiquement, l'utilisation des sources pulsées répétitives (excimère, YAG et $\mathrm{CO}_{2}-\mathrm{TEA}$ ) pour des problèmes de nettoyage et de préparation de surface. Les deux exemples abordés en détail sont : le nettoyage de moules métalliques d'injection par laser YAG et la préparation de surface par laser excimère de composites avant collage.
\end{abstract}

\section{DESCRIPTION DU PROJET «PRESAGE »}

Le projet PRESAGE (PREparation de Surface et nettoyAGE par laser) a pour objectifs : d'étudier la préparation de surface et le nettoyage par laser pulsé (excimère, YAG et $\mathrm{CO}_{2}$-TEA), de valider lintérêt technico-économique du procédé et de développer un moyen prototype, afin de répondre aux contraintes de productivité, économique par la diminution des coûts de fabrication et d'environnement par la suppression de l'utilisation de solvants et des rejets chimiques. Ce projet regroupe d'une part, des industriels exerçant dans des domaines différents :

- aéronautique : AEROSPATIALE et EUROCOPTER,

- automobile : RENAULT et PSA,

- agro-alimentaire : BSN emballages,

et d'autre part, des partenaires répartis comme suit :

- fabricant de sources lasers : QUANTEL (laser YAG) et SOPRA (laser excimère),

- centre de transfert de technologie laser : IREPA et CLAIRE,

- intégrateur : FABRICOM AUTOMATION,

- informatique industrielle : VOIR,

- universitaire : IRPHE (Institut de Recherche des Phénomènes Hors Equilibre) et Université Claude Bernard de Lyon.

Au travers des deux applications aéronautiques, à savoir : le nettoyage de moules métalliques d'injection (fabrication des pièces en composite) par laser YAG et la préparation de surface par laser excimère de composites (carbone-époxy) avant collage, les principales phases du projet ont été abordées :

- expression des besoins industriels,

- compréhension des phénomènes d'interaction laser-matière et détermination des paramètres de traitement,

- caractérisation des surfaces,

- analyse de l'impact sur l'environnement et de la toxicité

- première validation technico-économique à partir des essais sur des pièces réelles ou des

éprouvettes représentatives,

- réalisation d'un prototype,

- validation finale sur prototype.

Ce projet, d'une durée de trois ans et rentrant dans le cadre des Grands Projets Innovants, a été cofinancé par le Ministère de l'Industrie. 


\section{NETTOYAGE DE MOULES D'INJECTION PAR LASER YAG}

Les outillages de moulage par injection permettent de réaliser des pièces en matériaux composites de formes très complexes. Afin de pouvoir sortir les pièces ébauches, un démoulant est utilisé. Malgré cela, il faut d'une part éliminer les résidus de résine (époxy par exemple) subsistant à la surface ou dans les conduits d'amenée du moule métallique (acier ou aluminium), et d'autre part nettoyer ce dernier (élimination du film de démoulant, graisse, huile, ...). Ces opérations délicates (pour éviter d'endommager les surfaces) sont essentiellement réalisées de façon manuelle (grattoir, toile émeri, ...) et peuvent représenter entre 10 et $30 \%$ du cycle de fabrication d'une pièce selon sa complexité.

Les essais de nettoyage ont été réalisés sur un moule en acier recouvert d'une couche de résine époxy. Parmi les trois lasers sélectionnés initialement, le laser YAG fibré a été retenu pour réaliser un nettoyage de moule métallique, car il répond à tous les critères définis initialement :

- préservation de l'outillage,

- pas de dégagement de produits toxiques (décollement de la résine),

- fort rendement du nettoyage,

- facilité d'emploi (maniabilité et accessibilité) grâce à l'utilisation de la fibre optique.

Il permet, de plus, de réaliser un nettoyage complet du moule en une seule opération (élimination de la résine et du démoulant).Les essais sur le démonstrateur ont été réalisés en laissant le moule fixe et en balayant les zones à traiter avec le YAG fibré (Figure 1).

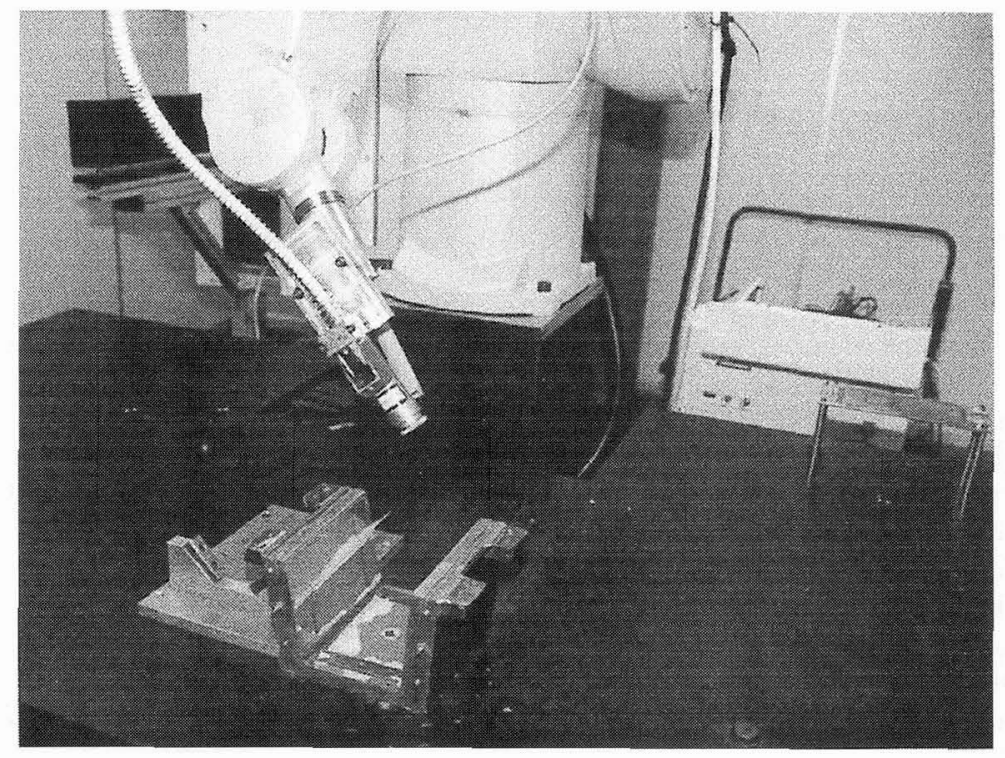

Figure 1 : Exemple de nettoyage de moule d’injection avec le démonstrateur

Un nettoyage complet du moule est obtenu pour des densités d'énergie de $200 \mathrm{~mJ} / \mathrm{cm}^{2}$ (inférieures au seuil d'ablation de la résine époxy : $320 \mathrm{~mJ} / \mathrm{cm}^{2}$ et au seuil d'endommagement du moule $: 1 \mathrm{~J} / \mathrm{cm}^{2}$ ). Un seul tir (Figure 2) permet de décoller la résine du moule sur une surface correspondant à la surface d'interaction. Le décollement de la résine se caractérise par un blanchiment de celle-ci. Une fois l'ensemble de la surface balayé, la pellicule de résine part d'elle-même. Il suffit de quelques minutes pour nettoyer complètement le moule. Les différents essais réalisés ont permis de montrer que le procédé est indépendant de l'épaisseur de résine (de quelques centièmes de $\mathrm{mm}$ à plusieurs $\mathrm{mm}$ ). 


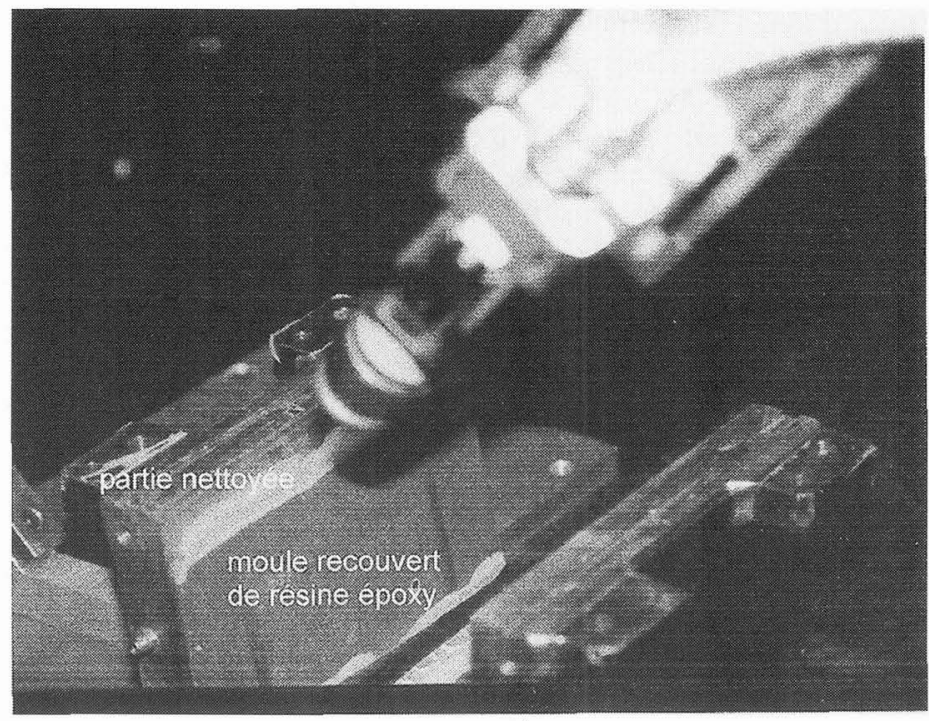

Figure 2 : Décollement de la résine de la surface du moule

\section{PREPARATION DE SURFACE AVANT COLLAGE DE MATERIAUX COMPOSITES}

Afin de permettre l'assemblage par collage d'éléments de structure en composite, il est nécessaire de réaliser une préparation de surface, pour d'une part, éliminer les multiples contaminants pouvant subsister à la surface ou dans la couche superficielle de résine (oxydation, démoulants, salissures diverses) et d'autre part, activer chimiquement la surface en introduisant des fonctions réactives. La méthode utilisée actuellement, le toilage manuel au papier abrasif, présente de nombreux inconvénients : manque de reproductibilité, car il s'agit d'une méthode manuelle, et utilisation de solvant avant ponçage pour le dégraissage des surfaces, et après ponçage pour éliminer les poussières de ponçage (Figure 3 ).
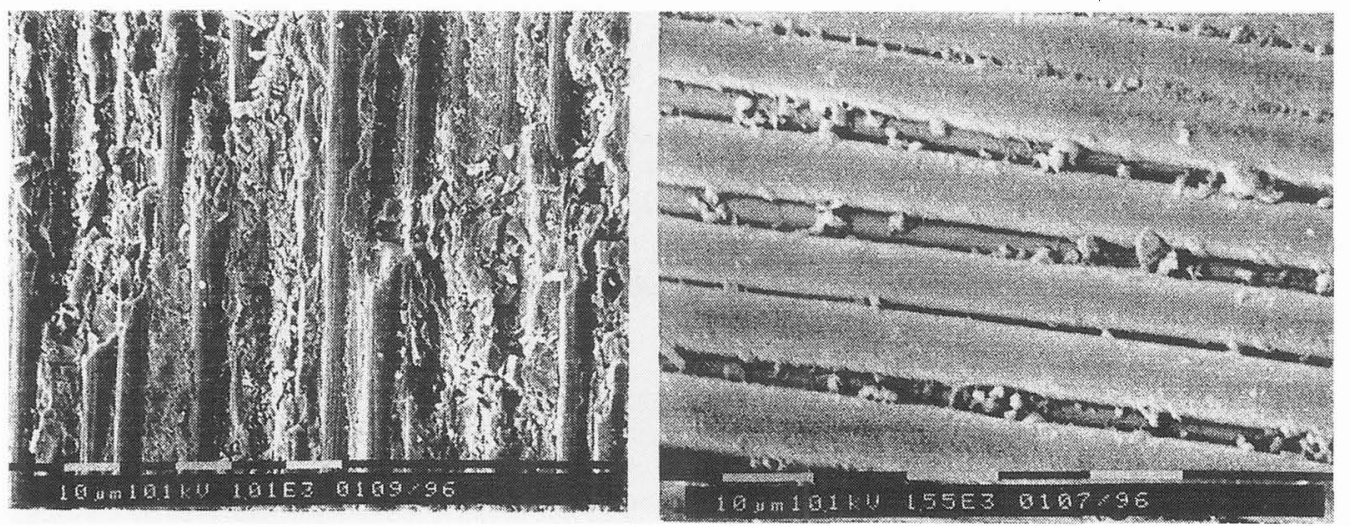

Figure 3 : Préparation de surface du composite carbone-époxy par ponçage (photographie de gauche) et par laser excimère (photographie de droite)

Le laser excimère $\mathrm{KrF}(248 \mathrm{~nm})$ a été retenu pour ce type de traitement, car il permet de nettoyer la surface (élimination des contaminants), d'activer la surface (chaque photon ayant une énergie suffisante pour briser de nombreuses liaisons atomiques), et d'ablater la résine en surface sans endommager les fibres. La densité d'énergie incidente est comprise entre 70 et $120 \mathrm{~mJ} / \mathrm{cm}^{2}$ et le nombre de tirs entre 5 et 100 . En fonction de la densité d'énergie incidente, il est possible de distinguer deux plages de traitements dans 
lesquelles les processus d'adhérence sont différents : en-dessous de $100 \mathrm{~mJ} / \mathrm{cm}^{2}$ (densité d'énergie inférieure au seuil d'ablation), l'adhérence est d'origine chimique (formation de nouvelles fonctions polaires à la surface), au-dessus de $100 \mathrm{~mJ} / \mathrm{cm}^{2}$ (densité d'énergie supérieure au seuil d'ablation), les fibres sont mises à nu ce qui permet à l'adhésif de s'ancrer mécaniquement autour de ces fibres (figure 3). Cette dernière configuration a été retenue, car elle permet d'obtenir une tenue au test de cisaillement (Figure 4) nettement supérieure aux autres traitements (manuel, plasma micro-ondes, :

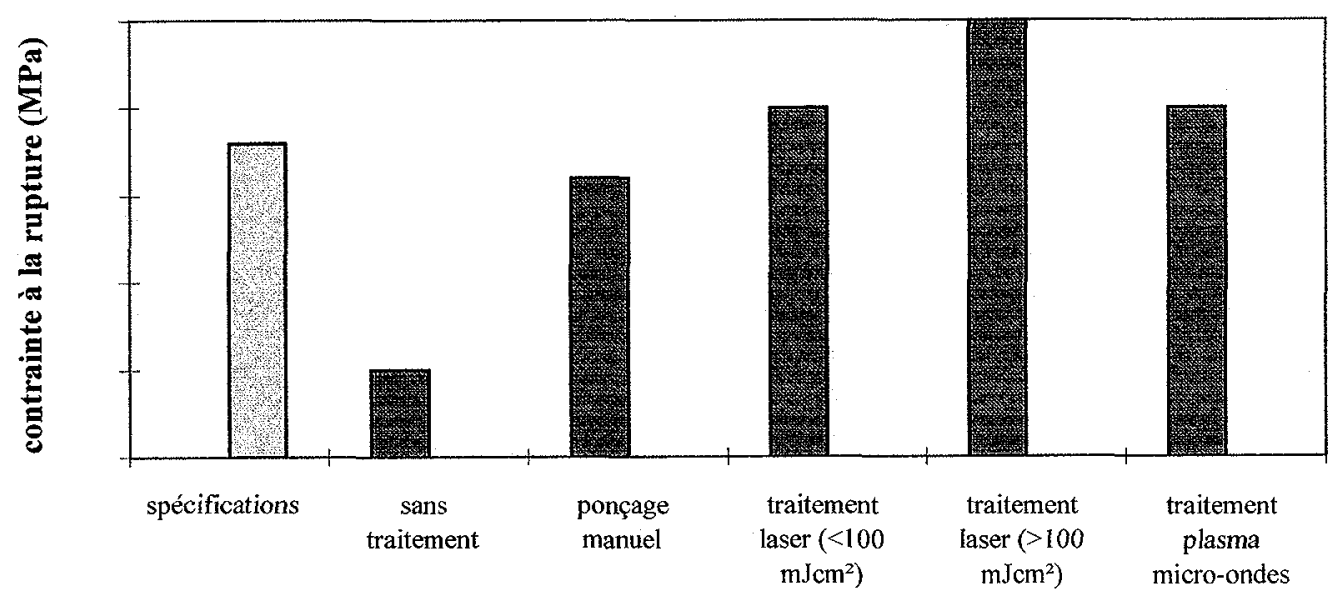

Figure 4 : Contraintes de cisaillement obtenues pour différents traitements

Le traitement obtenu est localisé (donc parfaitement adapté au collage de pièce de structure), reproductible et homogène. Cette technologie laser apporte des réponses positives à ces trois aspects :

- élimination de l'utilisation de solvant,

- augmentation des performances (contrainte à la rupture) de $20 \%$,

- vitesse multipliée par 4.

\section{CONCLUSION}

Le projet PRESAGE a permis à l'ensemble des partenaires d'évaluer techniquement et économiquement l'utilisation des sources lasers pulsés répétitifs $\left(\mathrm{CO}_{2}-\mathrm{TEA}, \mathrm{YAG}\right.$ et excimère) pour la préparation des surfaces avant collage de matériaux composites et le nettoyage de moules d'injection. Il existe actuellement un fort intérêt à court terme pour ces applications.

Après une étude préliminaire menée sur des moyens de laboratoire, la réalisation d'un démonstrateur (laser + robot) a permis de mettre en évidence les points :

- suppression complète de l'utilisation de solvant,

- préservation de l'outillage (nettoyage de moules),

- fort rendement en comparaison avec les procédés traditionnels,

- utilisation en mode manuel ou automatique,

- nette augmentation des performances des pièces traitées. 